et le titre individuel étant seul soumis, après les deux ans de rigueur, au timbre d'abonnement.

Il en résultera que l'on aura le bénéfice de la décision cidessus rappelée, prise par le Tribunal de Lille à la date du six juillet 1905 , et rapportée dans la solution du 10 avril 5906 (Voir sur ce point le deuxième supplément de Maguéro. Vo, Titres négociables, $\mathrm{n}^{0} 7$ ).

Rappelons enfin, que la taxe d'abonnement doit être suspendue pour les Sociétés improductives quand il y a deux ans d'improductivité.

Paul Bougault; Arocat à la Cour d'Appel de Lyon.

\section{DISJONCTEUR CARTER}

Parmi les nombreux perfectionnements réalisés dans l'appareillage électrique pour répondre aux problèmes toujours nouveaux posés par l'industrie moderne, il en est un qui mérite d'être signalé tout particulièrement, car l'appareil qui le réalise a sa place marquée dans toutes les stations centrales d'éclairage ou de traction.

Cet appareil est un disjoncteur qui résout le problème suivant:

$1^{-}$De ne pas pouvoir ètre fermé, s'il y a sur la ligne une intensité plus forte que celle que l'on a prévue.

$2^{\circ}$ De s'ouvrir lorsqu'il y a un court circuit ou un excès de déhit, même lorsque l'on maintient à la main la manette qui commande la fermeture.

On comprend de suite tout l'intérêt que présente un pareil dispositif; en effet, lorsqu'un disjoncteur à maxima fonctionne, la surintensité qui provoque le déclanchement est due généralement $\dot{a}$ une cause anormale dont on ignore souvent la provenance et la durée; il est donc essentiel d'avoir une impossibilité matérielle de fermer à nouveau le circuit tant que cette cause subsiste.

Ce disjoncteur se présente sous une forme assez différente de celles auxquelles les modèles existant nous ont habitués.

Tous les organes mécaniques sont enfermés dans une boite métallique d'où sortent, d'une part, l'axe du levier de commande, et, d'autre part, l'axe supportant les pièces de fixation des balais.

$\mathrm{Au}$-dessus des balais se trouvent deux jeux de pareétincelles formés, le premier par des touches en cuivre, et le second par des blocs de charbon.

Ces pare-étincelles sont établis de telle façon qu'à la rupture la séparation des différents contacts s'effectue dans l'ordre suivant : $1^{\circ}$ balais; $2^{\circ}$ pare-étincelles en cuivre; $3^{\circ}$ pare-étincelles en charbon. L'écartement de ces pareétincelles au moment du déclanchement est considérable et se produit d'une façon très brusque qui permet de rompre une intensité quelconque.

Sur le devant de l'appareil se trouve un bouton poussoir qui permet de provoquer le déclanchement à la main, sans avoir recours à la manette qui, placée sur le côté, ne'sert ainsi qu'à la fermeture de l'appareil.

Voyons maintenant par quel moyen sont obtenues les conditions de sécurité dont nous avons parlé plus haut. Le principe mécanique sur lequel repose cet appareil est très simple, et en voici la description schématique :

Soit $A B$ un levier qui commande d'une manière quelconque le disjoncteur en pivotant autour du point $A$. Cette commande, au lieu de se faire directement à la main, se fait par l'intermédiaire d'une chaîne $C$, qui passe sur un galet $G$ dont l'axe $S$ est fixe. Cette chaîne vient s'attacher à un levier $L M$, pivotant autour du point $L$, et mancuvré par la poignée $M$.

L'axe $S$ étant fixe, si l'on appuie sur la poignée $M$, on soulève le levier $A B$, lequel est ramené par le moyen d'un ressort $r$. On conçoit facilement que, dans ces conditions, à l'aide de la poignée $M$, l'on puisse faire manouvrer le disjoncteur qui est solidaire de $A B$.

Si maintenant le galet $G$ est supporté par un levier $O P$ pivotant autour de $O$, et maintenu fixe en $P$ par un cliquet $T$, on se rend facilement compte que, tant que le cliquet $T$ est maintenu dans la même position, l'axe $S$ est fixe, et la châine $C$, tendue, obéit à l'action de la poignée $M$. Mais si à un moment donné. le cliquet $T$ est dégagé, le levier $O P$ s'abaisse; l'axe $S$ n'étant plus fixe, la chaine n'est plus tendue, et le levier $A B$, n'obéissant plus à l'action de la poignée $M$, ne peut plus être soulevé.

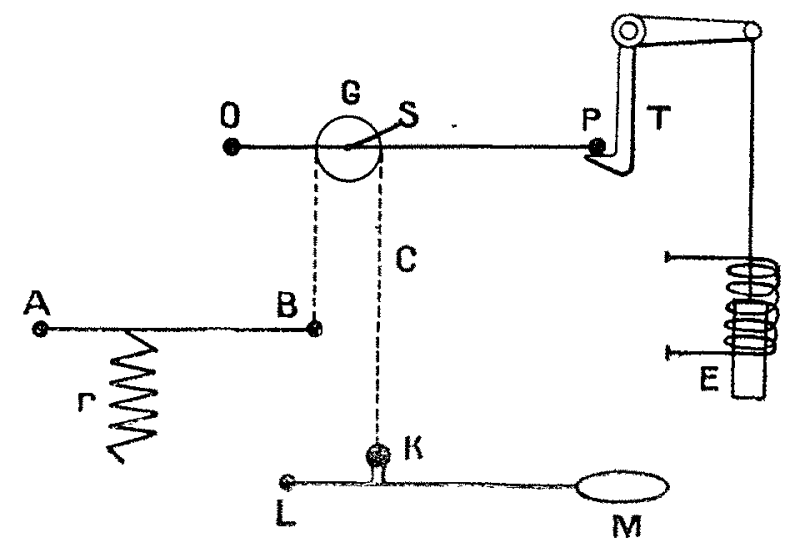

En somme, le procédé consiste à manœuvrer un appareil en prenant un point d'appui intermédiaire, lequel doit ètre fixe en temps normal, et mobile en cas d'anomalie.

Le soulèvement du cliquet $T$ est provoqué par un électro $E$ qui est, ou bien branché aux bornes du disjon. teur, ou bien mis dans le courant principal. Cet électro pent comprendre également à la fois les 2 en roulements, l'un el fil fin, l'autre en gros fil, pour réaliser certaines conditions que nous verrons plus loin.

Voyons maintenant le parti que l'on a tiré de cette idé dans la construction du disjoncteur.

Tout le mécanisme est renfermé dans une boîte mét:l. lique fermée, d'où le nom de "Carter » donné à ce disjoncteur. On retrouve dans ce mécanisme toutes les pièces dont nous venons de parler.

Le levier $A B$ est solidaire de l'axe sur lequel est fixí l'équipage mobile qui tient les balais de contact; la chaine est constituée par une forte chaine Galle; elle est attachée au levier $L M$ de telle sorte qu'au moment de la fermeture elle passe tout près de l'axe $\mathrm{L}$, ce qui donne pratiquement une tension considérable.

Cet effort que l'on obtient ainsi à la main est utilisé en vue de réaliser des contacts parfaits. Les bala's employés sont des balais doublement contre-coudés et les surfaces de contact se présentent parallèlement, ce qui permet d'avoir des contacts d'autant meilleurs que la pression est plus forte, à l'inverse de ce qui se produit avec les balais taillés en sifflet.

L'avantage qui résulte, en outre, d'une pareille dispo* sition, est de permettre l'emploi du même mêcanisme pour des appareils d'intensités très différentes, et jusqu'à des intensités très élevées, en changeant simplement la largeur et le nombre des balais.

L'intérieur du “Carter " comprend également la bobine qui sert au déclanchement, el cette bobine est conçue de telle façon qu'elle puisse servir à tous les types. Elle est com: posée d'un nombre uniforme de spires en gros fil, et il suffit de la shunter convenablement par des lames de maillechort, découpées à l'avance, pour ob̀tenir, sur un mème disjoncteur, le fonctionnement à une intensité quelconqu au-dessus de 50 ampères.

On peut obtenir ainsi un premier réglage, qui est fall une fois pour toutes et au commencement de la mise en 
service de l'appareil; un autre réglage est obtenu, en outre, par la pénétration plus ou moins grande du noyau dans l'intérieur du solénoïde ; cette marge de réglage est d'environ 10 pour 100 de l'intensité qui produit le déclanchement lorsque le noyau est à la position la plus élevée, c'est-à-dire la plus rapprochée du solénolide.

Ce déplacement est obtenu très facilement au moyen d'un index muni d'un bouton molleté, placé intérieurement sur le côté du carter et qui se déplace sur un petit cadran gradué de 100 à 140.

Ce réglage est extrêmement précis, et se maintient à moins de 4 pour 100 près du point obtenu une première fois. Cette précision est égrale à celle des appareils de mesure, et l'on est complètement maitre de la marge que l'on doit se laisser.

Ce disjoncteur, par la description qui en a été donnée jusqu'ici, se présente donc naturellement comme un disjoncteur à maxima. Nous allons voir comment, sans rien changer à la partie mécanique, il est possible d'en faire un disjoncteur à minima, ayant une précision égale à celle qu'il a comme disjoncteur à maxima, et pouvant aussi se régler.

Pour cela, autour de la bobine précédente on ajoute simplement un enroulement de fil fin, pris en dérivation sur les deux pôles extrêmes du disjoncteur. Prenons le cas où l'on veut obtenir un disjoncteur à minima polarisé.

Supposons que le noyau, pour être mis en action par l'intermédiaire du gros fil seul, ait besoin de $N$ ampères-tours. et supposons que le fil fin fournisse 9 N/10 ampères-tours.

Si les deux magnétismes s'ajoutent, dans le sens normal de marche, aussitôt que le gros fil produit $N / 10$ ampères tour's, le déclanchement aura lieu. Mais l'on a soin, pour le sens normal de fonctionnement, de connecter l'enroulement $\dot{a}$ fil fin, en sens inverse de l'enroulement en gros fil, si bien que les actions des deux magnétismes se retranchent le noyau sollicité en marche normale par $N-9 N / 10$ ampères tours seulement ne bougera pas.

Si le courant s'inverse, comme il ne change pas de sens dans la bobine en fil fin, les deux magnétismes s'ajoutent, et l'on a vu que le déclanchement a lieu lorsque le gros fil produit $N / 10$ ampères tours, c'est-à-dire le 1/10 de la marche normale. On a vu que le déplacement du noyau permet une marge de réglage allant de $N$ à 1,4 $N$; ceci représente une différence du 4 / 10 ampères tours, et comme le nombre des ampères tours ne varie pas dans la bobine en fil fin, il faudra, pour obtenir le déclanchement, faire passer dans le gros fil 5 N/10 ampères tours ; l'on obtient ainsi le déclanchement pour la moitié de l'intensité normale inversée.

On comprend aisément qu'il est facile, en changeant le nombre d'ampères tours de la bobine fll fin, de provoquer le déclanchement entre zéro et une intensité négative quelconque.

On peut donc employer ce disjoncteur pour batterie d'accumulateurs, en le faisant déclancher à une intensité pour la décharge et à une autre intensité pour la charge.

Ainsi, par cet aperçu rapide, l'on voit qu'il est facile d'utiliser ce disjoncteur dans presque tous les cas, et que sa place est marquée tout particulièrement partout où l'on désire avoir une sécurité absolue de fonctionnement.

Nous pouvons ajouter, à titre d'indication, qu'il a été utilisé pour la mise en parallèle, soit de deux dynamos, soit d'un dynamo et d'une batterie d'accumulateurs, et que, dans ces divers cas, l'appareil s'opposait au couplage tant que les sources d'énergie à coupler étaient à un voltage différent de plus de 5 volts.

Enfin, pour terminer, signalons que cet appareil est depuis quelques mois adopté par la marine nationale, et qu'il est rendu réglementaire sur tous les nouveaux bâtiments en construction.

L'avantage que présente le dispositif que nous venons

de décrire est qu'étant entièrement mécanique il peut être adapté à tout appareil devant remplir des conditions analogues de sécurité. C'est ainsi qu'il estutilisé non seulement pour les disjoncteurs à haute tension à rupture sous l'huile, mais aussi pour les appareils de démarrage ordinaires et les démarreurs régleurs genre contrôleur.

(L'Industrie des Tramways et Chemins de fer.)

\section{Decret du 17 actobre 1907 portant fixation des redevances pour l'occupation du domaine public par les entreprises de distribution d'énergie électrique.}

\section{Le Président de la République française,}

Sur le rapport du Ministre de l'Intérieur, du Ministre des Finances, du Ministre des Travaux publics, des Postes et des Télégraphes, et du Ministre de l'Agriculture;

Vu la loi du 15 juin 1906 sur les distributions d'énergie, et notamment l'article $18\left(7^{\circ}\right)$ portant qu'un règlement d'administration publique fixe les redevances dues à l'Etat, aux départements et aux communes, en raison de l'occupation du domaine public par les ouvrages des entreprises concétées ou munies de permission de voirie;

\section{Le Conseil d'Etat entendu,}

Décrète :

Article premier. - Les redevances pour l'occupation du domaine public par les ouvrages de transport d'énergie électrique alimentant les services publics assurés ou concédés par l'Etat, les départements et les communes, sont proportionnelles à la longueur des lignes, au nombre des supports, et à la surface du domaine public occupé; elles sont perçues, conformément au tarif ci-après, par l'Etat, le département et la commune au prorata de la longueur des voies empruntées, suivant que ces voies font partie du domaine public national, départemental ou communal :

\section{Situation
des emplacements du domane public occupé}

Paris..................

Communes de roo.ooo habitants et au-dessus Communes de 20.000 à roo.00o habitants... Communes de moins de 20.000 habitants.....

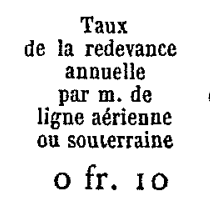

ofr. 02

ofr. oI

ofr. 005
Redevance chayutie support (Poteau ou pylone)

ro fr.
\end{abstract} par

$2 \mathrm{tr}$.

o fr. 50

o fr. 25 oste de transformateurs
et autres élablissements
anslogues analogues
(Minumum : $1 \mathrm{fr}$. par posite\} $25 \mathrm{tr}$.

Art. 2. - Les redevances pour l'occupation du domaine public par les ouvrages particuliers de transports et par les ouvrages de distribution, quel qu'en soit l'objet, sont fixées au double des taux prévus à l'article $\mathrm{r}^{\mathrm{er}} \mathrm{ci}$-dessus.

Art. 3. - Les redevances prévues aux articles I et 2 pour l'occupation du domaine public communal peuvent, en cas de distribution concédée, et en vertu d'une stipulation spéciale du cahier des charges, soit être réduites par l'autorité concédante, pour tenir compte des avantages particuliers réservés à la commune par l'acte de concession, soit être remplacées par des redevances proportionnelles au recettes brutes totales réalisées dans la commune, sans toutefois pouvoir dépasser les maxima fixés par le tarif ci-après, exprimés en pour cent des recettes:

$$
\text { Désignation des Communes }
$$

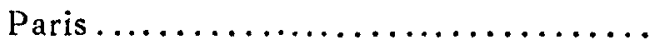

Communes de plus de 100.000 habitants

Communes de 20.000 à 100.000 habitants Communes ayant moins de 20.000 habit.

$\begin{array}{cc}\begin{array}{c}\text { Distribution } \\ \text { pour } \\ \text { l'éclairage }\end{array} & \begin{array}{c}\text { Distribution } \\ \text { pour tous } \\ \text { autres usages }\end{array} \\ 10 \% & 5 \% \\ 4 \% & 1,5 \\ 3 \% & 1, \% \\ 2 \% & 0,5\end{array}$

Les entrepreneurs de distributions établies en vertu de permis sions de voirie peuvent demander l'application du tarif maxi- 